\title{
Erratum to: The effects of antenatal corticosteroids therapy on very preterm infants after chorioamnionitis
}

\author{
Ken Miyazaki · Madoka Furuhashi · Kaoru Ishikawa Koji Tamakoshi · \\ Tomoaki Ikeda $\cdot$ Satoshi Kusuda $\cdot$ Masanori Fujimura
}

Published online: 21 February 2014

(C) Springer-Verlag Berlin Heidelberg 2014

\section{Erratum to: Arch Gynecol Obstet}

DOI 10.1007/s00404-013-3106-3

Inadvertently, two authors' names (Satoshi Kusuda, Masanori Fujimura) and their affiliations had been omitted in the author group of published article. The rearranged author group is given below.

Ken Miyazaki · Madoka Furuhashi · Kaoru Ishikawa · Koji Tamakoshi · Tomoaki Ikeda $\cdot$ Satoshi Kusuda $\cdot$ Masanori Fujimura

The online version of the original article can be found under doi:10.1007/s00404-013-3106-3.

K. Miyazaki $(\bowtie) \cdot$ M. Furuhashi

Department of Obstetrics and Gynecology, Japanese Red Cross

Nagoya Daiichi Hospital, 3-15 Michishita-cho, Nakamura-ku,

Nagoya 453-8511, Japan

e-mail: goldencaesar21@yahoo.co.jp

K. Ishikawa

Department of Endowed Chair for Regeneration of Medicine

in Kuwana District, Suzuka University of Medical Science,

Mie, Japan

K. Tamakoshi

Department of Nursing, Nagoya University Graduate School of

Medicine, Nagoya, Japan

T. Ikeda

Department of Obstetrics and Gynecology, Mie University

Graduate School of Medicine, Mie, Japan

\section{S. Kusuda}

Department of Neonatology, Tokyo Women's Medical University,

Tokyo, Japan

\section{Fujimura}

Department of Neonatology, Osaka Medical Center and Research Institute for Maternal and Child Health, Osaka, Japan

\section{S. Kusuda}

Department of Neonatology, Tokyo Women's Medical University, Tokyo, Japan

\section{Fujimura}

Department of Neonatology, Osaka Medical Center and Research Institute for Maternal and Child Health, Osaka, Japan 